\title{
High-Rate, High-Fidelity Entanglement of Qubits Across an Elementary Quantum Network
}

\author{
L. J. Stephenson, ${ }^{*}$ D. P. Nadlinger๑, ${ }^{*}$ B. C. Nichol, S. An๑, P. Drmota®, T. G. Ballance, ${ }^{\ddagger}$ K. Thirumalai, \\ J. F. Goodwin $\odot$, D. M. Lucas, and C. J. Ballance $\oplus^{\dagger}$ \\ Department of Physics, University of Oxford, Clarendon Laboratory, Parks Road, Oxford OX1 3PU, United Kingdom
}

(Received 3 December 2019; accepted 6 February 2020; published 16 March 2020)

\begin{abstract}
We demonstrate remote entanglement of trapped-ion qubits via a quantum-optical fiber link with fidelity and rate approaching those of local operations. Two ${ }^{88} \mathrm{Sr}^{+}$qubits are entangled via the polarization degree of freedom of two spontaneously emitted $422 \mathrm{~nm}$ photons which are coupled by high-numerical-aperture lenses into single-mode optical fibers and interfere on a beam splitter. A novel geometry allows highefficiency photon collection while maintaining unit fidelity for ion-photon entanglement. We generate heralded Bell pairs with fidelity $94 \%$ at an average rate $182 \mathrm{~s}^{-1}$ (success probability $2.18 \times 10^{-4}$ ).
\end{abstract}

DOI: 10.1103/PhysRevLett.124.110501

The ability to form logical connections between all quantum bits (qubits) of a quantum processor is a prerequisite for building a fault-tolerant universal device [1]. Trapped atomic ions have been identified as an excellent candidate qubit technology because they allow the implementation of single-qubit operations [2-4], twoqubit phonon-mediated gates $[3,4]$, and quantum memories $[5,6]$, all with high fidelity. However, the number of ions that can be reliably interfaced in a single trap is limited by the motional mode density, necessitating architectures with multiple trap zones each hosting comparatively few ions. Trap zones can be interfaced by physically shuttling qubits across centimeter-scale distances using electric fields [7], or by using photons to distribute entanglement over larger distances [8]. Photonic entanglement could also increase the connectivity of trapped-ion qubits via dynamically switchable fiber links [9], or allow the interfacing of different qubit platforms [10]. It also enables other quantum networking applications such as quantum key distribution, teleportation of quantum states, and "blind" quantum computing $[11,12]$. For ions, the entanglement rate is limited fundamentally only by the photon scattering rate ( $\sim 100 \mathrm{MHz})$, exceeding local multiqubit operation rates (motional gates [13] and shuttling [14,15]) at typical secular trap frequencies $(\sim 1 \mathrm{MHz})$. In practice, photonic entanglement rates have been far lower than this, limited principally by low photon collection efficiencies [16]; the highest previously reported rate for ions was $4.5 \mathrm{~s}^{-1}$, with $78 \%$ fidelity [17]. Faster rates have been achieved with nitrogen-vacancy centers $(39 \mathrm{~Hz})$ and quantum dots $(7.3 \mathrm{kHz})$, with fidelity $\approx 60 \%[18,19]$. Heralded entanglement of remote qubits with fidelity above $90 \%$ has not previously been reported for any physical systems at rates above a few milli-Hz [20-22].
In this Letter, we report the generation of entanglement between two qubits in separate ion traps at rates and fidelities approaching those of typical local (intratrap) operations, by swapping entanglement between photons emitted by the ions onto the ions themselves [23]. At these higher rates and fidelities, distillation procedures based on photonic entanglement [24] start to become a viable method for creating high quality entanglement across a scalable trapped-ion quantum computer.

A novel excitation scheme using ${ }^{88} \mathrm{Sr}^{+}$ions with photon collection perpendicular to the static applied magnetic field allows an increased rate over previous experiments [17], with polarization mixing maximally suppressed by coupling into a single mode optical fiber. In contrast to previous schemes using ${ }^{171} \mathrm{Yb}^{+}$, the collection geometry does not impede the use of beams parallel to the applied magnetic field. This allows standard $\sigma$-polarized optical pumping to be employed, thus permitting a wider choice of ion species and the straightforward initialization of multiple ion species in a single trap.

We collect photons from the spontaneous decay of the excited electronic state $\left|5 p P_{1 / 2}, m=+1 / 2\right\rangle$ of ${ }^{88} \mathrm{Sr}^{+}$, as shown in Fig. 1(a). Decays to the two states of the ground level $5 s S_{1 / 2}$ are associated with $\pi$ and $\sigma^{+}$polarized photons, forming an entangled ion-photon state given by

$$
|\psi\rangle=\sqrt{\frac{2}{3}}|\downarrow\rangle\left|\sigma^{+}\right\rangle+\sqrt{\frac{1}{3}}|\uparrow\rangle|\pi\rangle,
$$

where the weightings are due to the Clebsch-Gordan coefficients for each decay path, and the ion qubit states are labeled with $|\downarrow\rangle$ and $|\uparrow\rangle$. Perpendicular to the magnetic field axis, the emitted field from the $\pi$ decay has twice the intensity, and so for photons on the collection axis the ionphoton state is 


$$
|\psi\rangle=\frac{1}{\sqrt{2}}(|\downarrow\rangle|H\rangle+|\uparrow\rangle|V\rangle),
$$

where $\sigma^{+}$and $\pi$ have been relabeled $H$ and $V$ to emphasize that the two photon polarizations are both linear and orthogonal; note that this is a maximally entangled Bell state.

The nonorthogonality of the $\sigma^{+}$and $\pi$ emissions away from the collection axis would normally reduce the fidelity of the ion-photon entanglement at the high numerical apertures needed to maximize the photon collection efficiency [25]. However, with the chosen collection geometry, coupling into a single mode optical fiber rejects the nonorthogonal component of the $\sigma^{+}$emission, reducing the maximum possible collection efficiency but maintaining unit ion-photon Bell state fidelity independent of collection aperture [see Fig. 1(b)]. In contrast to other schemes, no photons of comparable wavelength are

(a)

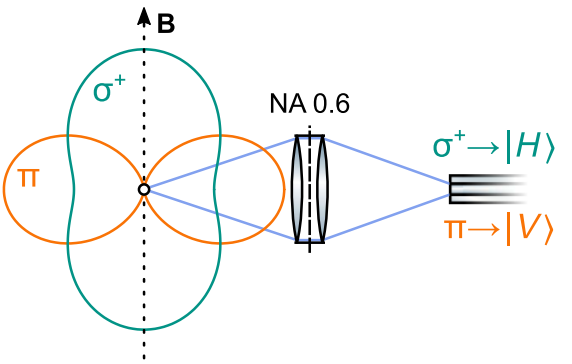

(b)

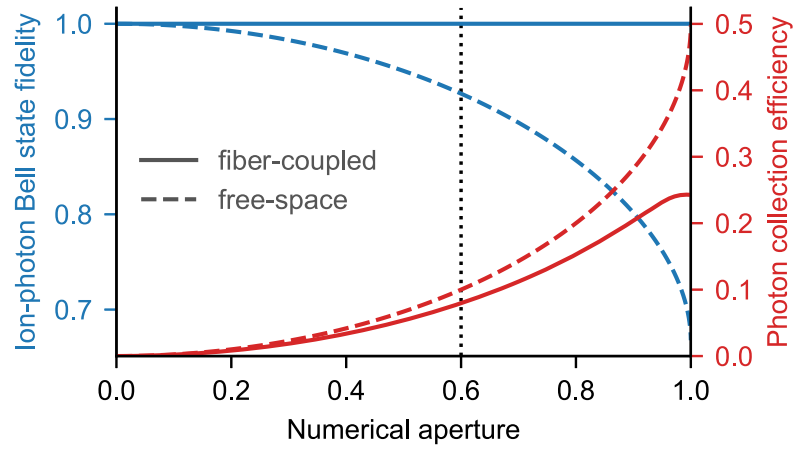

FIG. 1. (a) Intensity distribution of the light field for $\sigma^{+}$and $\pi$ decay channels, relative to the quantization axis set by the static magnetic field $\mathbf{B}$, and the branching fractions for each decay due to atomic selection rules. At the fiber input face, photons from $\sigma^{+}$ $(\pi)$ decay map to the $H(V)$ fiber polarization mode. (b) Maximum fraction of photons emitted by the ion that can be collected (red) and theoretical ion-photon entanglement fidelity (blue) versus collection optic numerical aperture. For free-space collection (dashed lines), polarization mixing leads to a loss in fidelity with increasing numerical aperture. To model the fiber-coupling (solid lines), we calculate the overlap of the light field with a Gaussian mode on the dashed plane in (a); a smaller fraction of the emission is collected, but the polarization mixing is completely suppressed. The vertical dashed line shows the NA 0.6 used in this Letter, where the fiber collection efficiency is $80 \%$ of that in free space. produced from undesired decay channels. This eliminates the need to filter out such photons [26], enabling higher rates to be achieved with our collection geometry and excitation scheme.

By collecting two such photons entangled with separated ions and erasing the which-path information from the photons, a projective measurement of the two-photon state in the Bell basis will herald the projection of the two ions into a corresponding Bell state [27].

In our experiment, ${ }^{88} \mathrm{Sr}^{+}$ions are trapped in two identical, high-optical-access, microfabricated surface traps [28] in two vacuum systems, designated "Alice" and "Bob," separated by $2 \mathrm{~m}$. In each system, a high-numericalaperture (NA 0.6) lens, aligned perpendicular to the applied magnetic field of $0.56 \mathrm{mT}$, couples single photons from the ion into an antireflection (AR) coated single-mode optical fiber. Non-polarization-maintaining (non-PM) fibers are used so as to introduce minimal differential phase between $H$ and $V$ photons (PM fibers introduce a large, temperaturesensitive, differential phase which would be difficult to control). A second objective (NA 0.3) images the ion through a slot in the trap onto a photomultiplier tube for fluorescence detection.

The relevant electronic structure of ${ }^{88} \mathrm{Sr}^{+}$is shown in Fig. 2. Ions are Doppler cooled with lasers at 422 and $1092 \mathrm{~nm}$. The Zeeman structure of the ground level is used to encode the "Zeeman" qubit: $\left|S_{1 / 2}, m=-1 / 2\right\rangle=:|\downarrow\rangle$ and $\left|S_{1 / 2}, m=+1 / 2\right\rangle=:|\uparrow\rangle$. We also define an "optical" qubit between the metastable level $\left|4 d D_{5 / 2}, m=-3 / 2\right\rangle=:|D\rangle$ and $|\downarrow\rangle$, and use a narrow linewidth laser at $674 \mathrm{~nm}$ to coherently transfer population between either of the Zeeman qubit states and $|D\rangle$, for ion state tomography. As $|D\rangle$ is outside the Doppler cooling cycle, it can also be used to shelve population from $|\uparrow\rangle$ to measure the ground state qubit by state-dependent fluorescence detection [29].

The experimental sequence for generating entangled photons is shown in Fig. 2. An optimized attempt section at rate $1 \mathrm{MHz}$, lasting at most $500 \mu \mathrm{s}$, is interleaved with $100 \mu$ s of Doppler cooling, until detection of an appropriate two-photon coincidence heralds the creation of ion-ion entanglement. (In single-ion/single-photon experiments, a single click of a chosen detector instead breaks this attempt loop and triggers the start of the analysis sequence.) The experimental sequence is controlled by an FPGA [30], incorporating the custom-optimized, precompiled section with decision branching in hardware, and just-in-time compiled sequences for qubit manipulations.

The projective measurement of the photons is performed with a partial Bell state analyzer, consisting of a 50:50 nonpolarizing beam splitter (NPBS) and polarizing beam splitters (PBSs) on each output arm. All four output channels are monitored by avalanche photodiodes (APDs, quantum efficiency 65\% [31]), as shown in Fig. 3. Spatial mode matching of the photons from each system at the NPBS is aided by recoupling the light into AR-coated single-mode 


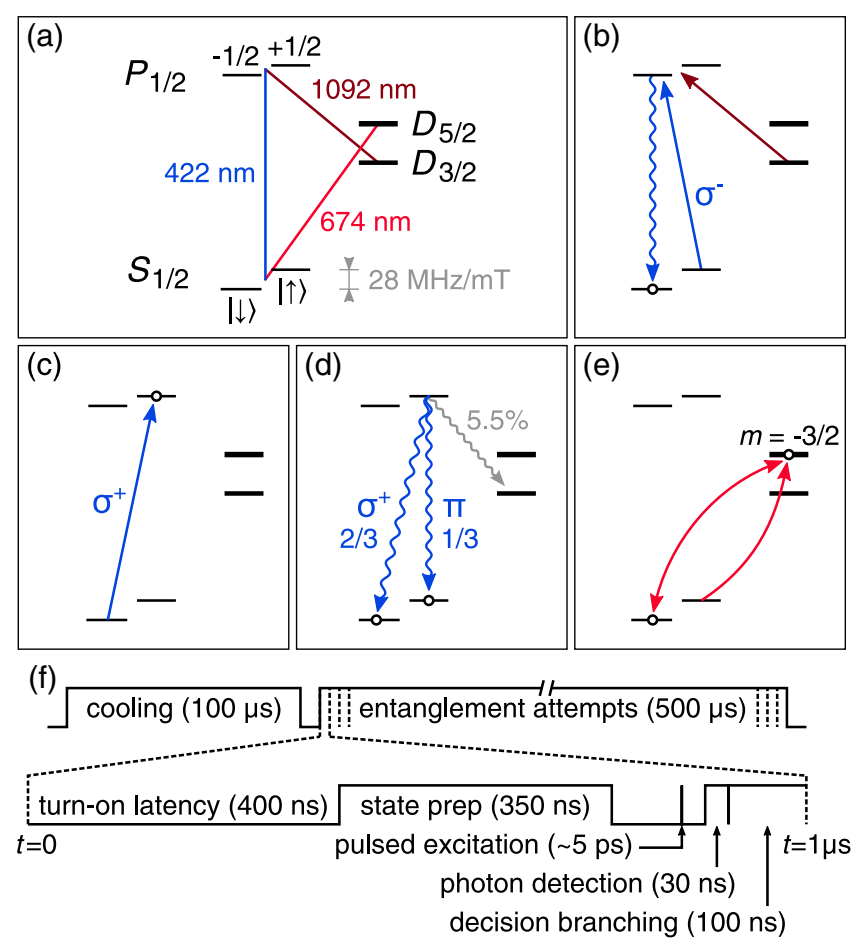

FIG. 2. (a) ${ }^{88} \mathrm{Sr}^{+}$level diagram (not to scale). (b) The initial state preparation consists of optical pumping on the $422 \mathrm{~nm}$ transition, with a repumper at $1092 \mathrm{~nm}$ to clear the $D_{3 / 2}$ level. (c) A single $\sim 5$ ps pulse from a frequency-doubled mode-locked Ti:sapphire laser coherently transfers the population to $P_{1 / 2}, m=+1 / 2$ with $\approx 97 \%$ probability. (d) The ion decays to a superposition of $|\downarrow\rangle$ and $|\uparrow\rangle$, emitting a photon whose polarization state is entangled with the state of the ion. Decays to the $D_{3 / 2}$ manifold occur with probability $5.5 \%$, but as the $1092 \mathrm{~nm}$ photons are not transmitted by the fiber, the only effect is to lower the overall rate. (e) Coherent manipulations are performed on the $674 \mathrm{~nm}$ transition to $|D\rangle$ in order to analyze the final ion qubit state. (f) Experimental sequence: the ions are Doppler cooled for $100 \mu \mathrm{s}$ before the attempt loop (lasting up to $500 \mu \mathrm{s}$ ) begins. The enlarged view shows a single attempt, with $\approx 400 \mathrm{~ns}$ of latency between state preparation turn-on signal (at $t=0$ ) and light arriving at the ion. State preparation $(\approx 350 \mathrm{~ns})$ is followed by a $100 \mathrm{~ns}$ delay to ensure that the beams are fully extinguished before the pulsed excitation. The $30 \mathrm{~ns}$ photon detection window begins $30 \mathrm{~ns}$ after the excitation pulse to allow for detector latency. A further $100 \mathrm{~ns}$ is required to decide whether to branch out of the attempt loop, in the event that a herald pattern is detected.

optical fibers before the APDs (coupling efficiency $\approx 90 \%$ ). The total click efficiency into all APDs is typically $2.1 \%$ and $2.4 \%$ for Alice and Bob, respectively. These measured efficiencies imply that the mode matching into the first fiber is $\gtrsim 50 \%$ of the theoretical optimum [8.0\%, Fig. 1(b)].

We first characterize the entanglement between the ion and emitted photon for each of the trap systems, using one detector in the apparatus shown in Fig. 3. We perform full tomography of the combined ion-photon state by independently rotating each qubit. Rotations of the ion state are

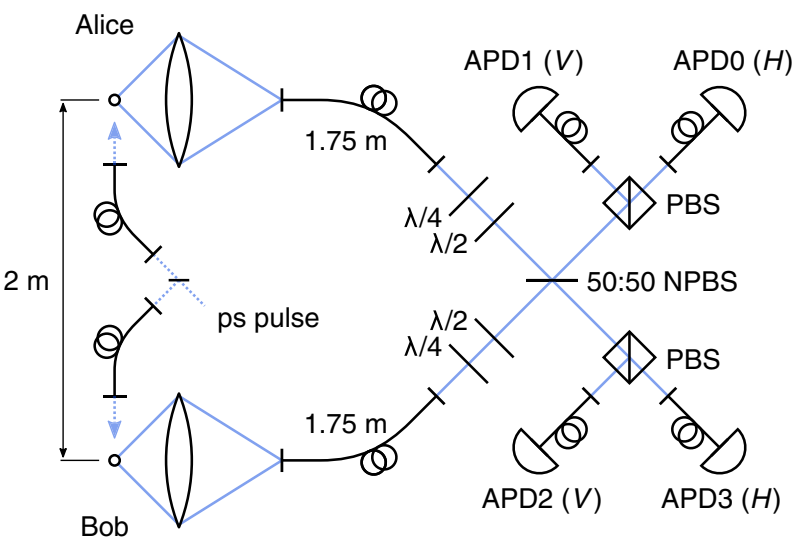

FIG. 3. Overview of apparatus. Single pulses from a frequencydoubled mode-locked laser are split to simultaneously excite ions in Alice and Bob. The spontaneously emitted photons are collected into single-mode non-PM fibers, with wave plates directly after the fibers to rotate the polarization. The photons are then overlapped on a 50:50 NPBS, and detected on singlemode-fiber-coupled APDs following PBSs. To minimize the polarization dependence of the NPBS, we use a small angle of incidence $\left(\approx 10^{\circ}\right)$. The same apparatus is used for single-ion/ single-photon experiments.

performed on the optical qubit using the $674 \mathrm{~nm}$ laser after mapping $|\uparrow\rangle$ to $|D\rangle$ with a $\pi$ pulse. Rotations of the photon state are performed using the wave plates in the Bell state analyzer. An overcomplete set of ion and photon measurements is used to characterize the entangled ion-photon state, and to calculate the maximum-likelihood estimate (MLE) of the composite density matrix. The density matrices obtained indicate a fidelity of 97.90(12)\% $(97.70(12) \%)$ with the maximally entangled state, at an average rate of $4.0 \times 10^{3} \mathrm{~s}^{-1}\left(5.7 \times 10^{3} \mathrm{~s}^{-1}\right)$ for the Alice (Bob) system.

Ion qubit rotation errors account for $\approx 0.6 \%$ of the total error, at $\approx 0.3 \%$ per rotation. We measure correlations of ion state with photon polarization of $P(\uparrow \mid V) \approx P(\downarrow \mid H) \approx$ 0.995 , which includes the error from one $\pi$ pulse on the ion qubit. This bounds the error due to all polarization mixing effects to $\lesssim 0.2 \%$. Excited state preparation errors (preparing $\left|P_{1 / 2}, m=-1 / 2\right\rangle$ instead of $\left|P_{1 / 2}, m=+1 / 2\right\rangle$ ) depend on the polarization impurity of both the optical pumping and pulsed excitation beams and are therefore suppressed. The remaining $1.4 \%$ error is attributed to ion qubit dephasing during the $60 \mu$ s delay between photon detection and tomography, and is expected to be due to noise in the applied magnetic field.

To entangle the two remote ion qubits, we erase the path information of photons entangled with each ion and subsequently project the ion-ion state via a destructive measurement on the photon polarizations. A coincidence detection on an appropriate pair of detectors heralds one of two Bell states: $\left|\Psi_{\text {photon }}^{+}\right\rangle:=(|V H\rangle+|H V\rangle) / \sqrt{2}$ if the detectors are on the same output port of the NPBS, and 
$\left|\Psi_{\text {photon }}^{-}\right\rangle:=(|V H\rangle-|H V\rangle) / \sqrt{2}$ if the detectors are on different output ports. Detection of $\left|\Psi_{\text {photon }}^{ \pm}\right\rangle$projects the ions correspondingly into $\left|\Psi_{\text {ion }}^{ \pm}\right\rangle:=\left(|\uparrow \downarrow\rangle \pm e^{i \phi}|\downarrow \uparrow\rangle\right) / \sqrt{2}$, where the phase $\phi$ is stable [32] and can be transformed to zero with local operations.

The probability of successfully heralding an entanglement event is given [17] by

$$
P=p_{\text {Bell }}\left[P_{\downarrow} P_{e} P_{S} \bar{P}_{\text {click }}\right]^{2}
$$

where $p_{\text {Bell }}=\frac{1}{2}$ because we have valid heralds only for two of the four possible two-photon Bell states, $P_{\downarrow} \approx 0.99$ is the probability of preparing the correct ground state before excitation, $P_{e} \approx 0.97$ is the population transferred to the excited state by the pulsed excitation beam, $P_{S} \approx 0.95$ is the probability of decaying to the $S_{1 / 2}$ ground states, and $\bar{P}_{\text {click }} \approx 0.023$ is the average probability of detecting a photon emitted by an ion. We measure $P=2.18 \times 10^{-4}$; given the average attempt rate (including Doppler cooling) of $833 \mathrm{kHz}, P$ yields a heralded ion-ion entanglement rate of $182 \mathrm{~s}^{-1}$.

After detection of a two-photon herald, we perform twoqubit tomography to verify the entangled state, using a series of single-qubit rotations and projective measurements [33]. The MLE ion-ion state is calculated for each of the four herald patterns individually, as shown in Fig. 4, indicating an average fidelity of $94.0(5) \%$ to the closest maximally entangled state [38].

The total ion-ion infidelity is dominated by errors in the ion-photon fidelity from each trap as described above, totaling $4.4 \%$, which includes errors in the ion-qubit rotations, ion dephasing, and polarization mixing effects. Additional infidelities include: the measured imperfections of the beam splitters in the Bell state analyzer, $\lesssim 0.17 \%$; temporal misalignment of the photons, $\lesssim 0.13 \%$; and dark counts, which contribute $\lesssim 0.05 \%$ despite a relatively high dark count rate of $\sim 60 \mathrm{~s}^{-1}$ per APD. The error due to mismatch of the photon modes at the NPBS is bounded by the measured fidelity to $<1.3(5) \%$, which is approximately consistent with independent measurements.

In summary, we have used a new combination of collection geometry and excitation scheme to demonstrate remote entanglement between two atomic ion qubits at much higher rates and fidelities than previously measured. The dominant infidelities arose from single-ion manipulations and spin decoherence, due to noise in the applied magnetic field and other known technical issues. An order of magnitude of rate improvement is feasible by reducing latencies and the duration of state preparation shown in Fig. 2. Further rate gains could exploit the quadratic dependence on detection efficiency $\bar{P}_{\text {click }}$ indicated by Eq. (2), by using detectors of higher quantum efficiency, improving the mode matching into the fibers or using higher numerical aperture lenses to increase collection efficiency. Significantly greater increases could in principle be realized by the use of a mirror close to the ion [39,40], or via the Purcell enhancement provided by an optical cavity [41-44]. Typical optical fiber losses at $422 \mathrm{~nm}$ are $30 \mathrm{~dB} \mathrm{~km}^{-1}$; frequency down conversion to the telecommunications $C$ band $(1550 \mathrm{~nm})$ [45] would allow the distribution of entanglement over much larger distances than in this experiment. The measured structure of the remote state produced is such that only two entangled pairs would be needed to distill a single remote entangled pair at or above $99 \%$ fidelity [46]. This would allow the photonic link to approach the performance of state-of-the-art local operations, enabling a variety of quantum networking applications.

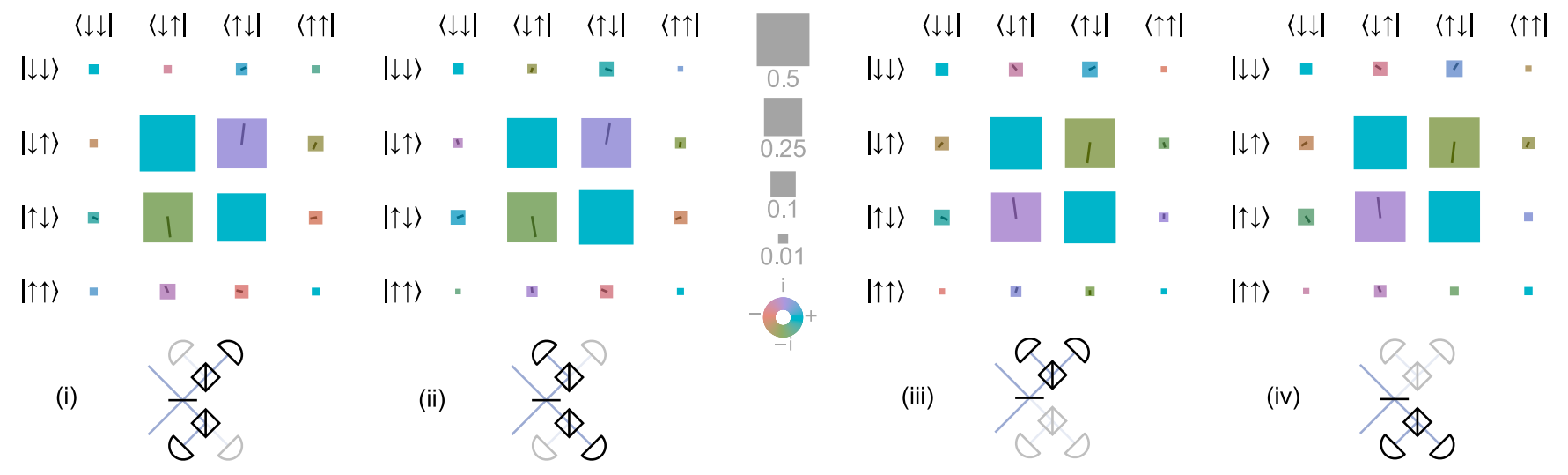

FIG. 4. The remote ion-ion density matrices and corresponding herald patterns (i)-(iv) as per the detector arrangement in Fig. 3. Detector clicks on opposing sides of the 50:50 beam splitter (i), (ii) herald projection into $\left|\Psi_{\text {ion }}^{-}\right\rangle$, while clicks on the same side (iii), (iv) herald $\left|\Psi_{\text {ion }}^{+}\right\rangle$. The average fidelity of all four patterns to the nearest maximally entangled state is $94.0(5) \%$, at a heralded rate of $182 \mathrm{~s}^{-1}$. (In this diagram the area of each square gives the magnitude of the matrix element, with the color representing the complex phase, according to the key shown. A "clock hand" also indicates the phase on the same color wheel. See the Supplemental Material [33] for numerical information.) 
We would like to thank Peter Maunz (Sandia National Laboratories) for supplying HOA2 ion traps, and the developers of ARTIQ [47]. B. C. N. and K. T. acknowledge funding from the U.K. National Physical Laboratory and the Defence Science and Technology Laboratory respectively. C. J. B. acknowledges support from a UKRI FL Fellowship, and is a Director of Oxford Ionics Ltd. This work was supported by the U.K. EPSRC "Networked Quantum Information Technology" Hub and the E.U. Quantum Technology Flagship Project AQTION (No. 820495).

*These authors contributed equally to this work. †chris.ballance@physics.ox.ac.uk Present address: ColdQuanta UK Ltd, Oxford.

[1] D. P. DiVincenzo, The physical implementation of quantum computation, Fortschr. Phys. 48, 771 (2000).

[2] T. P. Harty, D. T. C. Allcock, C. J. Ballance, L. Guidoni, H. A. Janacek, N. M. Linke, D. N. Stacey, and D. M. Lucas, High-Fidelity Preparation, Gates, Memory, and Readout of a Trapped-Ion Quantum Bit, Phys. Rev. Lett. 113, 220501 (2014).

[3] C. J. Ballance, T. P. Harty, N. M. Linke, M. A. Sepiol, and D. M. Lucas, High-Fidelity Quantum Logic Gates Using Trapped-Ion Hyperfine Qubits, Phys. Rev. Lett. 117, 060504 (2016).

[4] J. P. Gaebler, T. R. Tan, Y. Lin, Y. Wan, R. Bowler, A. C. Keith, S. Glancy, K. Coakley, E. Knill, D. Leibfried, and D. J. Wineland, High-Fidelity Universal Gate Set for ${ }^{9} \mathrm{Be}^{+}$ Ion Qubits, Phys. Rev. Lett. 117, 060505 (2016).

[5] Y. Wang, M. Um, J. Zhang, S. An, M. Lyu, J. N. Zhang, L. M. Duan, D. Yum, and K. Kim, Single-qubit quantum memory exceeding ten-minute coherence time, Nat. Photonics 11, 646 (2017).

[6] M. A. Sepiol, A. C. Hughes, J. E. Tarlton, D. P. Nadlinger, T. G. Ballance, C. J. Ballance, T. P. Harty, A. M. Steane, J. F. Goodwin, and D. M. Lucas, Probing Qubit Memory Errors at the Part-per-Million Level, Phys. Rev. Lett. 123, 110503 (2019).

[7] D. Kielpinski, C. Monroe, and D. J. Wineland, Architecture for a large-scale ion-trap quantum computer, Nature (London) 417, 709 (2002).

[8] C. Monroe and J. Kim, Scaling the ion trap quantum processor, Science 339, 1164 (2013).

[9] J. Kim, P. Maunz, T. Kim, J. Hussman, R. Noek, A. Mehta, C. Monroe, T. Ralph, and P. K. Lam, Modular Universal Scalable Ion-trap Quantum Computer (MUSIQC), AIP Conf. Proc. 1363, 190 (2011).

[10] H. M. Meyer, R. Stockill, M. Steiner, C. Le Gall, C. Matthiesen, E. Clarke, A. Ludwig, J. Reichel, M. Atatüre, and M. Köhl, Direct Photonic Coupling of a Semiconductor Quantum Dot and a Trapped Ion, Phys. Rev. Lett. 114, 123001 (2015).

[11] H. J. Kimble, The quantum internet, Nature (London) 453 , 1023 (2008).

[12] A. Broadbent, J. Fitzsimons, and E. Kashefi, Universal blind quantum computation, in Proceedings of the 50th Annual
IEEE Symposium on Foundations of Computer Science (2009), pp. 517-526.

[13] V. M. Schäfer, C. J. Ballance, K. Thirumalai, L. J. Stephenson, T. G. Ballance, A. M. Steane, and D. M. Lucas, Fast quantum logic gates with trapped-ion qubits, Nature (London) 555, 75 (2018).

[14] A. Walther, F. Ziesel, T. Ruster, S. T. Dawkins, K. Ott, M. Hettrich, K. Singer, F. Schmidt-Kaler, and U. Poschinger, Controlling Fast Transport of Cold Trapped Ions, Phys. Rev. Lett. 109, 080501 (2012).

[15] R. Bowler, J. Gaebler, Y. Lin, T. R. Tan, D. Hanneke, J. D. Jost, J. P. Home, D. Leibfried, and D. J. Wineland, Coherent Diabatic Ion Transport and Separation in a Multizone Trap Array, Phys. Rev. Lett. 109, 080502 (2012).

[16] C. Monroe, R. Raussendorf, A. Ruthven, K. R. Brown, P. Maunz, L.-M. Duan, and J. Kim, Large-scale modular quantum-computer architecture with atomic memory and photonic interconnects, Phys. Rev. A 89, 022317 (2014).

[17] D. Hucul, I. V. Inlek, G. Vittorini, C. Crocker, S. Debnath, S. M. Clark, and C. Monroe, Modular entanglement of atomic qubits using photons and phonons, Nat. Phys. 11, 37 (2015).

[18] P. C. Humphreys, N. Kalb, J. P. J. Morits, R. N. Schouten, R. F. L. Vermeulen, D. J. Twitchen, M. Markham, and R. Hanson, Deterministic delivery of remote entanglement on a quantum network, Nature (London) 558, 268 (2018).

[19] R. Stockill, M. J. Stanley, L. Huthmacher, E. Clarke, M. Hugues, A. J. Miller, C. Matthiesen, C. Le Gall, and M. Atatüre, Phase-Tuned Entangled State Generation between Distant Spin Qubits, Phys. Rev. Lett. 119, 010503 (2017).

[20] M. Lettner, M. Mücke, S. Riedl, C. Vo, C. Hahn, S. Baur, J. Bochmann, S. Ritter, S. Dürr, and G. Rempe, Remote Entanglement between a Single Atom and a Bose-Einstein Condensate, Phys. Rev. Lett. 106, 210503 (2011).

[21] S. Ritter, C. Nölleke, C. Hahn, A. Reiserer, A. Neuzner, M. Uphoff, M. Mücke, E. Figueroa, J. Bochmann, and G. Rempe, An elementary quantum network of single atoms in optical cavities, Nature (London) 484, 195 (2012).

[22] B. Hensen, H. Bernien, A. E. Dréau, A. Reiserer, N. Kalb, M. S. Blok, J. Ruitenberg, R. F. L. Vermeulen, R. N. Schouten, C. Abellán, W. Amaya, V. Pruneri, M. W. Mitchell, M. Markham, D. J. Twitchen, D. Elkouss, S. Wehner, T. H. Taminiau, and R. Hanson, Loophole-free Bell inequality violation using electron spins separated by 1.3 kilometres, Nature (London) 526, 682 (2015).

[23] M. Źukowski, A. Zeilinger, M. A. Horne, and A. K. Ekert, Event-Ready-Detectors Bell Experiment Via Entanglement Swapping, Phys. Rev. Lett. 71, 4287 (1993).

[24] R. Nigmatullin, C. J. Ballance, N. D. Beaudrap, and S. C. Benjamin, Minimally complex ion traps as modules for quantum communication and computing, New J. Phys. 18, 103028 (2016).

[25] B. B. Blinov, D. L. Moehring, L. M. Duan, and C. Monroe, Observation of entanglement between a single trapped atom and a single photon, Nature (London) 428, 153 (2004).

[26] D. L. Moehring, P. Maunz, S. Olmschenk, K. C. Younge, D. N. Matsukevich, L.-M. Duan, and C. Monroe, Entanglement of single-atom quantum bits at a distance, Nature (London) 449, 68 (2007). 
[27] C. Simon and W. T. M. Irvine, Robust Long-Distance Entanglement and a Loophole-Free Bell Test with Ions and Photons, Phys. Rev. Lett. 91, 110405 (2003).

[28] Sandia National Laboratories HOA2.

[29] A. H. Myerson, D. J. Szwer, S. C. Webster, D. T. C. Allcock, M. J. Curtis, G. Imreh, J. A. Sherman, D. N. Stacey, A. M. Steane, and D. M. Lucas, High-Fidelity Readout of Trapped-Ion Qubits, Phys. Rev. Lett. 100, 200502 (2008).

[30] ARTIQ Sinara hardware, https://m-labs.hk/experimentcontrol/sinara-core/.

[31] Laser Components COUNT BLUE, quantum efficiency measured at $422 \mathrm{~nm}$.

[32] L. J. Stephenson, Entanglement between nodes of a quantum network, D.Phil. thesis, University of Oxford, 2019.

[33] See Supplemental Material at http://link.aps.org/ supplemental/10.1103/PhysRevLett.124.110501 for a more detailed discussion of methods and data analysis, which includes Refs. [34-37].

[34] J. D. Wong-Campos, K. G. Johnson, B. Neyenhuis, J. Mizrahi, and C. Monroe, High-resolution adaptive imaging of a single atom, Nat. Photonics 10, 606 (2016).

[35] J. Rehacek, Z. Hradil, E. Knill, and A. I. Lvovsky, Diluted maximum-likelihood algorithm for quantum tomography, Phys. Rev. A 75, 042108 (2007).

[36] P. Faist and R. Renner, Practical and Reliable Error Bars in Quantum Tomography, Phys. Rev. Lett. 117, 010404 (2016).

[37] P. Faist, The tomographer project, v5.4, 2018.

[38] P. Badziąg, M. Horodecki, P. Horodecki, and R. Horodecki, Local environment can enhance fidelity of quantum teleportation, Phys. Rev. A 62, 012311 (2000).
[39] G. Shu, N. Kurz, M. R. Dietrich, and B. B. Blinov, Efficient fluorescence collection from trapped ions with an integrated spherical mirror, Phys. Rev. A 81, 042321 (2010).

[40] M. Fischer, M. Bader, R. Maiwald, A. Golla, M. Sondermann, and G. Leuchs, Efficient saturation of an ion in free space, Appl. Phys. B 117, 797 (2014).

[41] J. I. Cirac, P. Zoller, H. J. Kimble, and H. Mabuchi, Quantum State Transfer and Entanglement Distribution among Distant Nodes in a Quantum Network, Phys. Rev. Lett. 78, 3221 (1997).

[42] T. Kim, P. Maunz, and J. Kim, Efficient collection of single photons emitted from a trapped ion into a single-mode fiber for scalable quantum-information processing, Phys. Rev. A 84, 063423 (2011).

[43] A. Stute, B. Casabone, P. Schindler, T. Monz, P. O. Schmidt, B. Brandstätter, T.E. Northup, and R. Blatt, Tunable ionphoton entanglement in an optical cavity, Nature (London) 485, 482 (2012).

[44] A. Stute, B. Casabone, B. Brandstätter, K. Friebe, T. E. Northup, and R. Blatt, Quantum-state transfer from an ion to a photon, Nat. Photonics 7, 219 (2013).

[45] T. A. Wright, R. J. A. Francis-Jones, C. B. E. Gawith, J. N. Becker, P. M. Ledingham, P. G. R. Smith, J. Nunn, P. J. Mosley, B. Brecht, and I. A. Walmsley, Two-Way Photonic Interface for Linking the $\mathrm{Sr}^{+}$Transition at $422 \mathrm{~nm}$ to the Telecommunication C Band, Phys. Rev. Applied 10, 044012 (2018).

[46] S. Benjamin (private communication).

[47] S. Bourdeauducq et al., m-labs/artiq: 4.0 (Version 4.0), 2018. 